an association of NAFLD with ICP has not been previously evalu-

Insurance Generosity and Inflammatory Bowel Disease: Does Higher Patient Cost-Sharing Result in Suboptimal Medication Habits and Inferior Clinical Outcomes?

Lindsay Anne Sceats ${ }^{1}$, Cindy Kin, Amber Trickey, Maria Polyakova and M. Kate Bundorf

${ }^{1}$ Stanford University School of Medicine

OBJECTIVES/SPECIFIC AIMS: Our primary objectives were to examine the impact of biologic cost sharing on 1) adherence to biologics and 2) persistence on biologics in inflammatory bowel disease (IBD) patients. Our secondary objective was to assess the effect of biologic cost sharing on clinical IBD outcomes, including rates of hospitalization, abdominal surgery, and corticosteroid treatment. METHODS/STUDY POPULATION: This retrospective cohort analysis used a national insurance claims database (Optum Clinformatics DataMart) to assess adult IBD patients enrolled in medium or large private insurance plans from 2007-2016. Patients were followed for one year of continuous enrollment after their index biologic claim. We assessed adherence to biologic medications (medication possession ratio $>0.8$ ) dependent on patient cost sharing, as measured by an employer-plan's average out-of-pocket biologic medication cost. We also examined the effects of patient cost sharing for biologics on need for hospitalization, abdominal surgery, or corticosteroid treatment. We used multivariate logistic regression models adjusting for clinical and demographic characteristics. We estimated the effect of cost sharing on biologic therapy persistence using repeated measures proportional hazard survival models. RESULTS/ANTICIPATED RESULTS: We identified 2,193 adult IBD patients who initiated biologic therapy and met study criteria (Crohn's disease $66.1 \%$ vs. ulcerative colitis $24.9 \%$, mean age 40.8 years, mean Charlson index 0.50 ). Median [IQR] out-of-pocket cost per 30-day biologic prescription was $\$ 62$ [\$34 - \$157]. 66.9\% of patients were adherent to biologic therapy. Higher out-of-pocket costs for biologics were associated with increased odds of nonadherence; patients with ulcerative colitis were more price-responsive than patients with Crohn's disease or indeterminate colitis (Figure 1). However, higher out-of-pocket biologic costs were not associated with increased odds of all-cause or IBD-related hospitalization, IBD-related surgery, or corticosteroid prescriptions for IBD flares. Patients whose out-of-pocket costs were less than $\$ 10$ per 30 -day biologic prescription persisted on biologic therapy for significantly longer than patients who paid $>\$ 10$ (Figure 2). DISCUSSION/SIGNIFICANCE OF IMPACT: Nonadherence to biologics increases when IBD patients face higher out-of-pocket costs, particularly for ulcerative colitis patients. However, this is not associated with worse clinical outcomes. Patients with cost-sharing $<\$ 10$ persisted on biologics longer than patients whose cost sharing exceeded $\$ 10$.

3555

Intrahepatic Cholestasis of Pregnancy (ICP) is associated with higher prevalence of NAFLD: a case-control study. Erica Monrose ${ }^{1}$, Andres Ramirez Zamudio ${ }^{1}$, Alaina Aristide ${ }^{1}$, Reema Navalurkar ${ }^{1}$, Rashi Bedekar ${ }^{1}$, Lauren Ferrara ${ }^{1}$ and Tatyana Kushner ${ }^{1}$

${ }^{1}$ Icahn School of Medicine at Mount Sinai

OBJECTIVES/SPECIFIC AIMS: Intrahepatic Cholestasis of Pregnancy (ICP) is the most common liver disease unique to pregnancy. Progression of non-alcoholic fatty liver disease (NAFLD) has been linked to the dysregulation of bile acid homeostasis. However, ated. We evaluated the association between ICP and NAFLD and associated metabolic risk factors, including obesity, dyslipidemia, hypertension, and diabetes. METHODS/STUDY POPULATION: A single-center, retrospective case-control study was conducted between January and December 2017 in a primarily Latina population in a New York City health system with a high prevalence of ICP, $2.53 \%$ of all pregnancies (compared to $0.32 \%$ nationally). Pearson's chi-square or Fisher's exact test and Wilcoxon rank-sum tests were performed to evaluate association of ICP with categorical variables and continuous variables, respectively. Unadjusted odds ratios (OR) with $95 \%$ confidence intervals $(95 \% \mathrm{CI})$ were calculated in comparison with the control group for clinically significant outcomes. RESULTS/ANTICIPATED RESULTS: 149 pregnancies complicated by ICP were identified from electronic medical records; 200 controls were matched by delivery year. Hispanic women were more likely to be diagnosed with ICP than non-Hispanic women (OR 1.90, 95\% CI 1.87-3.03). ICP and control patients were similar for: median age (OR 1.02, 95\% CI.99-1.06), nulliparity (OR.79, 95\% CI.48-1.30) and prevalence of hepatitis C (OR 1.35, 95\% CI.08-21.67). In regards to metabolic risk factors, ICP patients and control patients were similar in prevalence of obesity (body mass index $>30 \mathrm{~kg} / \mathrm{m}^{\wedge} 2$; OR 1.01 , 95\% CI.62-1.61), hemoglobin A1c >5.5\% (OR.80, 95\% CI.34-1.9), total cholesterol $>200 \mathrm{mg} / \mathrm{dL}$ (OR 4.15, 95\% CI.83-20.84), and prevalence of hypertension (OR.69, 95\% CI.31-1.52). Median bile acid levels were $30.6 \mu \mathrm{mol} / \mathrm{L}$ (IQR 11.6, 32.7) in the ICP group. ICP patients had higher median alanine aminotransferase (ALT) (32 vs. $16 \mathrm{U} / \mathrm{L}$, $\mathrm{p}<.0001$ ), alkaline phosphatase (181 vs $128 \mathrm{U} / \mathrm{L}, \mathrm{p}<.0001)$ and total bilirubin levels $(0.5$ vs $0.35, \mathrm{p}<.0001)$ compared to controls. ICP patients were more likely to have ALT levels $>50 \mathrm{U} / \mathrm{L}$ (2 times the upper limit of normal; OR 3.22, 95\% CI 1.48-7.03). ICP patients were significantly more likely to have a history of biliary disease (OR 3.29, 95\% CI 1.39-7.80). ICP patients were more likely to have evidence of steatosis on liver imaging (OR 4.69, 95\% CI 1.68-13.12) than non-ICP patients. When evaluating a diagnosis of NAFLD based on ICD-10 code or evidence of steatosis on liver imaging, ICP patients were significantly more likely to have a diagnosis of NAFLD than controls (OR 5.7, 95\% CI 2.08-15.65). DISCUSSION/ SIGNIFICANCE OF IMPACT: ICP appears to be associated with NAFLD independently of metabolic risk factors such as obesity, dyslipidemia, hypertension, and diabetes, suggesting a direct link between NAFLD and ICP. If findings are confirmed, ICP patients, especially those with elevated ALT, would benefit from screening for NAFLD and linkage to liver specialty care postpartum.

3478

\section{Knowledge, Attitudes and Practices on End-of-Life Care among Healthcare Professionals in a Community Hospital in Puerto Rico}

Deana Hallman ${ }^{1}$ and Ivonne Jimenez

${ }^{1}$ University of Puerto Rico-Medical Sciences Campus

OBJECTIVES/SPECIFIC AIMS: The study aims to determine the baseline knowledge, attitudes and practices of the professionals (i.e., doctors and nurses) that provide the end-of-life care at an acute community hospital. By obtaining information on what is known, what is believed and what is done by doctors and nurses in endof-life care, we can identify needs, problems, barriers and possible solutions. METHODS/STUDY POPULATION:. Develop a questionnaire in Spanish addressing knowledge, attitudes and practices about end-of-life care in an acute hospital, define the survey objectives, and develop the survey protocol. o To validate the pertinence 